\title{
Lymphangiogenesis in cervical cancer evaluated by expression of the VEGF-C gene in clinical stage IB-IIIB
}

\author{
Magdalena Franc ${ }^{1}$, Agata Kachel-Flis², Bogdan Michalski ${ }^{3}$, Anna Fila-Daniłow ${ }^{4}$, Urszula Mazurek \\ Mateusz Michalski ${ }^{5}$, Anna Michalska ${ }^{6}$, Ilona Kuczerawy ${ }^{1}$, Violetta Skrzypulec-Plinta ${ }^{7}$
}

${ }^{1}$ Medical Department, Medical University of Silesia in Katowice, Poland

2Department of Oncology, St. Leszczynski Memorial Hospital in Katowice, Poland

${ }^{3}$ Department of Gynecological Oncology, Women School of Health, Medical University of Silesia in Katowice, Poland

${ }^{4}$ Department of Medical Genetics, Medical University of Silesia in Katowice, Poland

${ }^{5}$ Department of Gynecological Oncology, Gynecology and Obstetrics, Regional Railway Hospital in Katowice, Poland

${ }^{6}$ Department of Ophthalmology, Medical University of Silesia in Katowice, Poland

${ }^{7}$ Department of Woman School of Health, Medical University of Silesia in Katowice, Poland

\begin{abstract}
Introduction: The aim of the present study was to evaluate the profile of VEGF-C gene expression in particular stages of cervical cancer (IB-IIIB) and to estimate the correlation between VEGF-C mRNA quantity profile and clinical stage.

Material and methods: Material for molecular analysis consisted of cervical cancer tissue specimens collected from 38 women (10, 15, 13 cases were classified as IB, IIB and IIIB, respectively). The control group was composed of normal cervical tissues collected from 10 women who underwent hysterectomy for non-oncological reasons. The number of VEGF-C mRNA copies in particular groups was estimated by the reverse transcription quantitative polymerase chain reaction (RT-qPCR) method.

Results: In the control group the average number of mRNA copies was $134 \pm 36$ (median: 106), in a group with stage IB it was $16077 \pm 7090$ (median: 580), for stage IIB - $35019 \pm 8945$ (median: 40 870). The highest number of mRNA VEGF-C copies was derived in a group of patients with cervical cancer of stage IIIB. The average quantity was $56155 \pm 12$ 470, whereas median 55 981. A statistically significantly higher level of VEGF-C gene expression was disclosed in cervical cancer specimens with stage IIB and IIIB than in the control group. In stage IIIB, the VEGF-C gene expression was significantly higher than in specimens derived from individuals in stage IB.

Conclusions: In squamous cell carcinoma of the uterine cervix of stage IB-IIIB genes involved in lymphangiogenesis, especially VEGF-C, are expressed, which expression increases as the clinical stage of cervical cancer is higher.
\end{abstract}

Key words: cervical cancer, lymphangiogenesis, VEGF-C, metastases.

\section{Introduction}

Clinical observation reveals that the very first feature disclosing cervical cancer metastasis, as well as the majority of solid tumor metastasis, is the presence of cancer cells in regional lymph nodes. Malignant neoplastic tumors occupy regional nymph nodes by a few mechanisms such as intraluminal invasion of tumors lymphatic vessels or invasion of peritumoral lymphatic vessels accompanied by intensive lymphangiogenesis [1]. Molecular index of tumor cells transformation while acquiring invasive phenotype is an increasing expression of prolymphangiogenic and pro-angiogenic factors [2].

For the majority of solid tumors, including cervical cancer, the first aim of a metastatic process is regional lymph nodes, so attempting to clarify the lymphangio- genic process on a molecular level, including mean signalization paths, may provide valuable information about cancerogenesis, especially about mechanisms related to tumor progression.

The metastatic process consists of a few well-defined stages. First of them is the selection of cellular clone unsusceptible to anti-growth signals, growth factors self-production, uncontrolled proliferation, capability of avoiding apoptosis. The clone of these cells spreads reaching 1-2 $\mathrm{mm}$ diameter, until oxygen and nutritional supply gained from molecular diffusion stops being sufficient. Then, hypoxic environment stimulates angiogenesis, which is the second stage. The next stage is stromal invasion and then migration to low-resistant lymphatic vessels and veins. Groups of neoplastic cells detach from primary tumor forming cancer embolus in 
lymphatic vessels and veins so they can enter the circulatory system and in the last stage they reach target organs [3].

Among many molecules involved in lymphangiogenesis, proteins from the vascular endothelial growth factor family play a significant role, from which family vascular endothelial growth factor C (VEGF-C) plays an essential role by its receptor VEGFR-3, and less influential VEGF-D $[4,5]$. Both factors are substrates for VEGFR-3 but they also bind with VEGFR-2, which is a signal transducer for angiogenesis [6, 7]. Although expression of all 3 types of VEGF receptors during embryogenesis is significant for circular system development, after birth and in pathology VEGF-C expression is limited to lymphatic endothelium [8].

Vascular endothelial growth factor $C$ is produced in the cell as a precursor which transforms to mature ligand after proteolysis in the extracellular space. The vascular endothelial growth factor $C$ precursor $(61 \mathrm{kDa})$ is built of main homologous domain containing cysteine motives characteristic of the VEGF family, amine end of propeptide containing a signal sequence and carboxyl end of propeptide [9].

During synthesis 2 VEGF precursor molecules link by its opposite ends by sulfate bond and non-covalent bond forming dimers. As a result of extracellular secretion and containing amine and carboxyl end propeptide removing, a mature molecule of VEGF is formed (21 kDa) [9].

Vascular endothelial growth factor $C$ maturing process gives rise to increased affinity for VEGFR-2 and VEGFR-3 receptors. Precursor VEGF molecules also bind with VEGFR-3, however, proteolysis increases its affinity almost 400 times. It is assumed that synthesizing VEGF-C as a precursor molecule prevents from unnecessary angiogenic effect on VEGFR-2 receptor path and allows to conduct the signal by VEGFR-3 receptor. In this case proteolysis may play a regulating role for VEGF-C function [10].

The biological effect of $V E G F-C$, highly specific for lymphatic endothelial cells, is to stimulate processes such as proliferation, survival, migration, vessels permeability regulation. The mature form of $V E G F-C$ inducts proliferation, survival and migration by VEGFR-3 receptor and vessels permeability regulation by VEGFR-2 receptor $[6,9]$. Unlike VEGF, VEGF-C expression does not seem to be regulated by hypoxia but increases as a response to proinflammatory cytokines suggesting a significant $V E G F-C$ role in diseases with inflammatory background [6].

Regarding that VEGF-C expression was noticed in many human malignant tumors and that for majority of them disseminating to lymphatic nodes is the first stage of metastasizing, a significant role of VEGF-C in initiating metastasis is postulated $[5,6,11,12]$.

\section{Material and methods}

\section{Patients and materials}

Biopsy samples of uterine cervix tissue were obtained from 38 women (aged 45-70 years), in whom squamous cell carcinoma of the uterine cervix was diagnosed at the Department of Gynecological Oncology, Gynecology and Obstetrics, Regional Railway Hospital in Katowice. For further molecular studies, we selected 10,14, 13 tissue specimens with stage IB, IIB, IIIB, respectively. Specimens were with either $\mathrm{G} 1$ or $\mathrm{G} 2$ stage.

A control group was formed by tissues taken from 10 women at the age of 45-70, with neither histopathological nor oncological changes, who underwent hysterectomy for non-oncological reasons. In every case, two samples were taken from every patient, from the exact location of a histopathological change. Normal architecture of control tissues was confirmed by histopathological examination. Complete clinical, histologic and biologic data were available for each patient. All patients granted consent to collection and use of their biologic material. The study was carried out in compliance with the Code of Ethics of the World Medical Association (Declaration of Helsinki) and Polish regulations. It was approved by the Bioethics Committee of the Medical University of Silesia.

Histopathological evaluation was performed at the Department of Pathology of the Regional Railway Hospital in Katowice and at the Department of Histology, Jagiellonian University, Medical College in Krakow. Molecular analysis was performed at the Department of Molecular Biology, Medical University of Silesia in Katowice.

\section{RNA extraction}

The tissue samples of uterine cervix were immediately frozen in liquid nitrogen after resection and then stored at $-80^{\circ} \mathrm{C}$ until assayed. Total RNA was isolated from all frozen tissue samples with the use of TRIZOL ${ }^{\circledR}$ reagent according to the supplier's procedures (Invitrogen Life Technologies, USA). Quality of RNA extracts was checked electrophoretically using $1 \%$ agarose gel stained with ethidium bromide. The results were analyzed and registered using gel documentation system 1D Bas-Sys (Biotech-Fisher). In the case of RNA extracts contaminated by genomic DNA, the latter was removed by DNase I, RNasefree (MBI Fermentas, Burlington, Canada) according to manufacturer's instructions. The concentration of total RNA was determined by spectrophotometric measurement using the GeneQuant II RNA/DNA Calculator (Pharmacia Biotech, Cambridge, UK). The absorbance ratios $\left(A_{260 / 280}\right)$ for RNA samples were between 1.8 and 2.0. 
Tab. I. Characteristics of primers used in the real-time reverse transcription quantitative polymerase chain reaction (RT-qPCR) assay

\begin{tabular}{lcccc}
\hline Gene name & Sense primer & Antisense primer & $\begin{array}{c}\text { Access } \\
\text { number }\end{array}$ & $\begin{array}{c}\text { Amplicon } \\
\text { size (bp) }\end{array}$ \\
\hline VEGF-C & 5'-TGCCGATGCATGTCTAAACT-3' & 5'-TGACAGGTCTCTTCATCCAGC-3' & GI: 23273809 & 251 \\
\hline GAPDH & 5'-GAAGGTGAAGGTCGGAGTC-3' & 5'-GAAGATGGTGATGGGATTTC-3' & GI: 83641890 & 226 \\
\hline ACTB & 5'TCACCCACACTGTGCCCATCTACGA-3' & 5'CAGCGGAACCGCTCATTGCCAATGG-3' & GI: 5016088 \\
\hline
\end{tabular}

bp - base pare, GI - GenBank accession number (http://www.ncbi.nlm.nih.gov/)

\section{Design of specific primers}

Very specific primers were used in the real-time reverse transcription quantitative polymerase chain reaction (RT-qPCR). The sequences of these primers are presented in Table I.

The primers for VEGF-C have been described by Niki et al. [13]. We have used the same primers for glyceraldehyde-3-phosphate dehydrogenase (GAPDH) and actin beta $(A C T B)$ as those used in TaqMan GAPDH Control Reagent Kit and TaqMan PCR Reagent Kit (Applied Biosystems, USA). The specificity of all these primers was checked using BLAST program (http://blast.ncbi. nlm.nih.gov).

\section{One-step real-time $R T-q P C R$}

Transcriptional activity of all genes was analyzed using one-step real-time RT-qPCR method. This was performed using the Opticon ${ }^{\mathrm{TM}}$ DNA Engine Sequence Detector (MJ Research Inc., Watertown, MA) and the QuantiTect $^{T M}$ SYBR $^{\oplus}$ Green RT-PCR kit (Qiagen, Valencia, CA), as recommended by the manufacturer. Each reaction contained $25 \mu \mathrm{l}$ of the $2 \mathrm{X}$ QuantiTect SYBR Green RT-PCR Master Mix, $0.1 \mu \mathrm{M}$ of forward primers and $0.1 \mu \mathrm{M}$ of reverse primers, $0.5 \mu$ of the QuantiTect RT Mix, 100 ng of total RNA and RNase-free water to complete the whole up to $50 \mu \mathrm{l}$. The thermal cycling parameters were the following: $30 \mathrm{~min}$ at $48^{\circ} \mathrm{C}$ for reverse transcription, $10 \mathrm{~min}$ at $95^{\circ} \mathrm{C}$ for deactivation reverse transcriptases and activation HotStarTaq DNA Polymerase, then 40 cycles composed of $10 \mathrm{~s}$ at $95^{\circ} \mathrm{C}$ for melting, $60 \mathrm{~s}$ at $60^{\circ} \mathrm{C}$ for annealing and $45 \mathrm{~s}$ at $72^{\circ} \mathrm{C}$ for extending phases of the PCR reaction.

During the $q P C R$ stage, polymerase amplifies the target sequence, which creates the PCR products. The SYBR Green dye then binds to each new copy of double-stranded DNA. As the qPCR progresses, more amplicons are created. Since the SYBR Green dye binds to all double-stranded DNA, the result is an increase in fluorescence intensity proportionate to the amount of qPCR product produced. RT-qPCR was performed 3 times for each primer set and the mean was used for relative quantification. Moreover, in each case a negative control (without RNA) and a positive control (with mRNA GAPDH) were added. At the same time, for each analysis, mRNA for GAPDH was amplified as an endog- enous RNA control. The amount of VEGF-C mRNA was inferred from the $C_{T}$ and by using the standard curve for $A C T B$ standards performed during the same experiment ( $\beta$-actin Control Reagent Kit; Applied Biosystems, USA).

\section{Quality of RT-qPCR products}

Each product of RT-qPCR reaction was examined by melting temperature profile immediately after finishing PCR, in order to confirm the specificity of the amplification and absence of primer dimers (Fig. 1). Melt analysis followed by heating up to $95^{\circ} \mathrm{C}$ at a rate of $0.1^{\circ} \mathrm{C} / \mathrm{s}$ with concomitant monitoring the decrease in fluorescence. The rate of decrease of fluorescence intensity $(-d F / d T)$ was plotted to obtain $T_{m}$ value for each melting curve. The temperature was optimum when it gave the maximum reading for a specific product and a non-specific product could no longer be detected. In addition, RT-PCR products were run on $6 \%$ polyacrylamide gels and were visualized with silver salts.

\section{Statistical analysis}

The results were statistically analyzed using Excel and Statistica 8.0 software. The mean, standard error of the mean (SEM), median, minimal and maximal values were assessed for measurement data (number of VEGF-C mRNA). The Kolmogorov-Smirnov test was used to estimate normality of the distribution. Due to absence of distribution's normal features, nonparametric methods were used. A statistically significant difference between groups was evaluated with the Mann-Whitney $U$ test. The level $p<0.05$ was assumed as statistically significant.

\section{Results}

In the control group mean quantity of VEGF-C mRNA copies was $134 \pm 36$ (median: 106), in a group with stage IB it was $16077 \pm 7090$ (median: 580), for stage IIB - $35019 \pm 8945$ (median: 40 870). The highest quantity of VEGF-C mRNA copies was derived in a group of patients with cervical cancer of stage IIIB. The average quantity was $56155 \pm 12$ 470, whereas median 55981 . VEGF-C mRNA copies quantity was counted for $1 \mu \mathrm{g}$ of a total mRNA (Table II). 
A

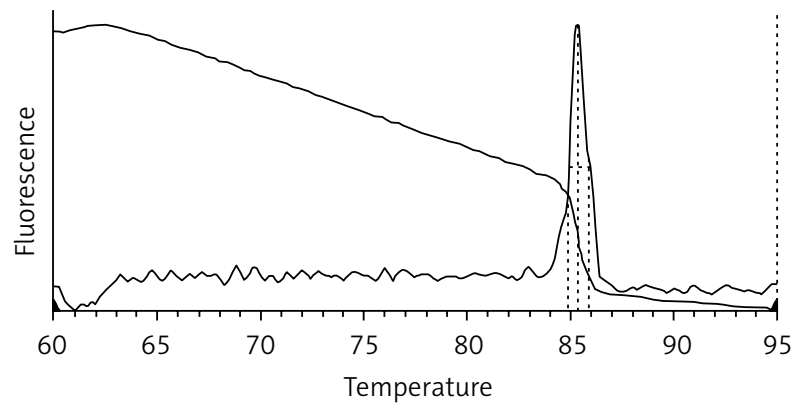

C

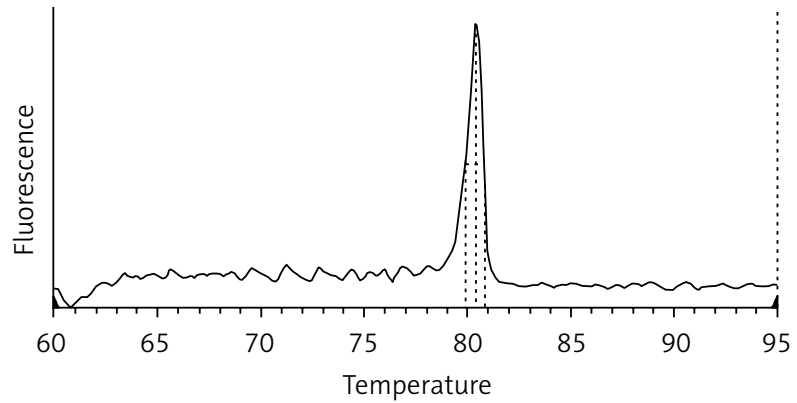

B

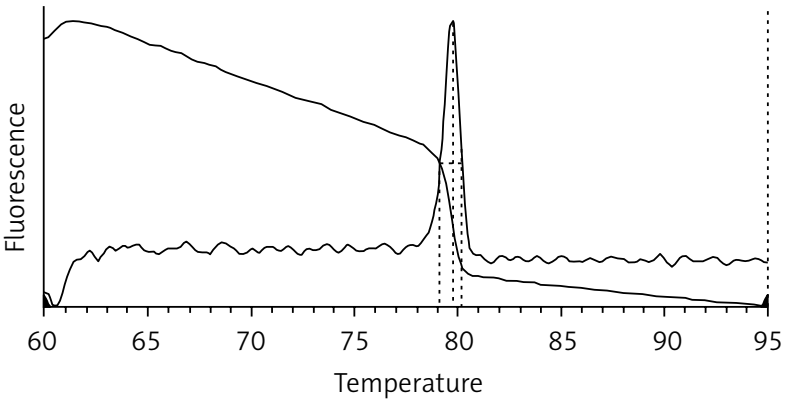

Fig. 1. The melting temperature curve of each analyzed gene: A) $A C T B, T_{m}=85.4^{\circ} \mathrm{C}$; B) GAPDH, $T_{m}=79.8^{\circ} \mathrm{C}$; C) VEGF-C, $\mathrm{T}_{\mathrm{m}}=80.4^{\circ} \mathrm{C} . \mathrm{T}_{\mathrm{m}}-$ melting temperature

Tab. II. Comparison of vascular endothelial growth factor C (VEGF-C) mRNA copies quantity in the control group and in groups with cervical cancer of stage IB-IIIB (Mann-Whitney's $U$ test)

\begin{tabular}{|c|c|c|c|c|c|}
\hline \multirow{2}{*}{ Gene } & & \multirow{2}{*}{ Group K } & \multicolumn{3}{|c|}{ Cervical cancer - stage } \\
\hline & & & IB & IIB & IIIB \\
\hline & $n$ & 10 & 10 & 15 & 13 \\
\hline & Mean & 134 & 16077 & 35019 & 56155 \\
\hline \multirow[t]{5}{*}{ VEGF-C } & SEM & 36 & 7090 & 8945 & 12470 \\
\hline & Median & 106 & 580 & 40870 & 55981 \\
\hline & Min-Max & $13-379$ & $12-59786$ & $105-95605$ & 259-125 124 \\
\hline & & $\mathrm{K}$ & 0.1124 & $<0.001$ & $<0.001$ \\
\hline & & & 1 & 0.1204 & 0.0131 \\
\hline \multicolumn{3}{|c|}{ Comparison of groups $-p$ (Mann-Whitney's $U$ test) } & & II & 0.1020 \\
\hline
\end{tabular}

Comparing levels of VEGF-C gene expression in particular groups, its higher notes in advanced stages have been noticed, however the difference was not always statistically significant (Fig. 2). A statistically significantly higher level of VEGF-C gene expression was disclosed in tissue specimens with stage IIB, IIIB cervical cancer, comparing to the control group. In stage IIIB, $V E G F-C$ gene expression was significantly higher than in tissue specimens with stage IB (Fig. 2).

The number of VEGF-C mRNA copies was counted for $1 \mu \mathrm{g}$ of a total mRNA. Although constant increase in VEGF-C mRNA copies with more advanced stage has been noted, a statistically significant difference between control group specimens and specimens with cancer of stage IB was not confirmed. However, a sta- tistically significant difference in the number of VEGF-C mRNA copies between stage IIB and IIIB was confirmed (Table II).

\section{Conclusions}

A lot of attention is paid to lymphangiogenesis and its meaning for neoplastic formation. It is also the subject of many studies. Profiles of prolymphangiogenic genes expression in particular tumors are different. The prognostic value of expression intensity of some molecules involved in lymphangiogenesis can be found in the literature [14]. It includes papillary carcinoma of the thyroid where the value of VEGF-C and MMP-2 as new, independent, prognostic factors for patients with 
lymph nodes metastasis has been confirmed. The increased quantity of VEGF-C and MMP-2 identified patients with metastatic dissemination to lymph nodes, poor prognosis and aggressive disease trial [15].

In the presented material, VEGF-C expression in all examined cervical cancers stages has been revealed. The difference in VEGF-C mRNA quantity between the control group and groups with stage IIB, IIIB and also between group IB and IIIB has been noticed. Similar results were obtained by $\mathrm{Ma}$ et al. [16] and Sotiropoulou et al. [17]. VEGF-C expression increase as the clinical stage is higher, although differences in particular groups are not always significant. These results concur with results presented by Jach et al. [18] and also with Japanese authors: Mitsuhasshi et al., where correlation between VEGF-C quantity and clinical stage (FIGO) has been revealed, however, serous level of $V E G F-C$ in squamous carcinoma of the uterine cervix with immunoenzyme techniques was assessed. Mitsuhasshi et al. [19], however, did not report any correlation between VEGF-C quantity and metastatic dissemination to lymph nodes. Nevertheless, Hashimoto and Codama's study [20] proved a correlation between VEGF-C quantity and lymph nodes metastasis and also depth of stromal invasion.

Many authors emphasize the correlation between VEGF-C, VEGF-D, VEGFR-3 quantity and metastatic dissemination to lymph nodes, tissue invasion and aggressive clinical trial of the neoplastic disease. This type of correlation has been noticed in colon cancer [13], endometrium [21], ovarian cancer [22] and breast cancer [23], however, some of studies do not concur with these observations $[24,25]$ or present opposite data [15]. This indicates a complex character of interaction of cytokines: VEGF-C/VEGF-D/VEGFR-3, which in particular tumors can be different.

Gombos and $\mathrm{Xu}$ [1] reported a correlation between VEGF-C expression quantity (assessed by immunohistochemistry) and density of lymphatic vessels (LVD) in the peritumoral area in the early stage of cervical cancer. In this study there was also documented prognostic value of LVD parameter regarding total survival of patients with cervical cancer. Research based on immunohistochemistry about VEGF-C and VEGFR-3 expression in cervical cancer, conducted by Schi and Xi [26], proved a positive correlation between VEGF-C and VEGFR-3 expression. It allows to assume that in squamous carcinoma of the uterine cervix there is a positive feedback between proangiogenic ligands expression and expression of its receptors. The opposite phenomenon is observed in normal tissues, where an increased cytokine level is accompanied by decreased receptors density, as a sign of cellular defense from excessive receptors stimulation effects ("down regulation" phenomenon) [27-29].

In Van Trappen's study [30], VEGF-C, VEGF-D and VEGFR-3 expression was evaluated by QRT-PCR method

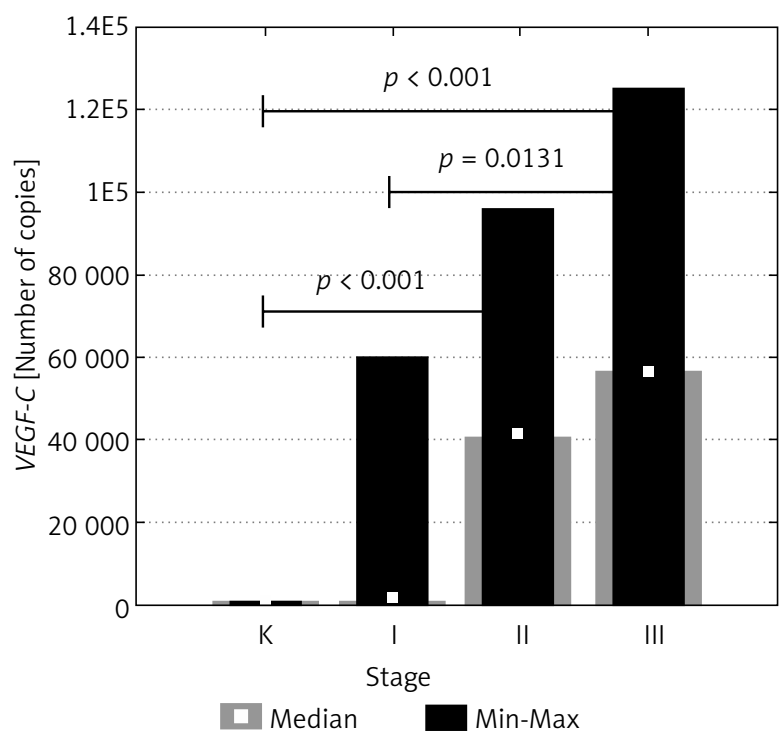

Fig. 2. VEGF-C mRNA copies quantity in the control group and in groups of patients in stage IB-IIIB

among patients presenting preinvasive cervical lesions and cervical cancer. Significant difference in VEGF-C, $V E G F-D, V E G F R-3$ expression levels between groups CINI, CINII and group CINIII and lack of difference between CINIII and squamous carcinoma of the uterine cervix was noted.

In squamous carcinoma of the uterine cervix of clinical stage IB-IIIB genes involved in lymphangiogenesis, especially VEGF-C, are being expressed. VEGF-C expression increase as the clinical stage is higher.

\section{Disclosure}

Authors report no conflict of interest.

\section{References}

1. Gombos Z, Xu X, Chu CS, et al. Peritumoral lymphatic vessel density and vascular endothelial growth factor $C$ expression in early stage squamous cell carcinoma of the uterine cervix. Clin Cancer Res 2005; 23 : 8364-8371.

2. Banyś A, Orchel J, Mazurek U, et al. Aktywność transkrypcyjna genów szlaków sygnalizacyjnych receptora VEGFR-2 w raku szyjki macicy wyznaczona techniką mikromacierzy oligonukleotydowych. Prz Menopauzalny 2009; 8: 72-75

3. Langley RR, Fidler IJ. Tumor cell-organ microenvironment interactions in the pathogenesis of cancer metastasis. Endocrin Rev 2007; 28: 297-321.

4. Detmar M, Hirakawa S. The Formation of Lymphatic Vessels and Its Importance in the Setting of Malignancy. J Exp Med 2002; 196: 713-718.

5. He Y, Rajantie I, Pajusola K, et al. Vascular endothelial cell growth factor receptor 3-mediated activation of lymphatic endothelium is crucial for tumor cell entry and spread via lymphatic vessels. Cancer Res 2005; 65: 4739-4746.

6. Jussila L, Alitalo K. Vascular growth factors and lymphangiogenesis. Physiol Rev 2002; 82: 673-700.

7. Roberts N, Kloos B, Cassella M, et al. Inhibition of VEGFR-3 activation with the antagonistic antibody more potently suppresses lymph node 
and distant metastases than inactivation of VEGFR-2. Cancer Res 2006; 66: 2650-2657.

8. Tammela T, Heckman C, Alitalo K. Lymphangiogenesis and metastasis. Am Assoc Cancer Res Educ Book 2005; 2005: 67-72.

9. Sundar SS, Ganesan TS. Role of limphangiogenesis in cancer. J Clin Oncol 2007; 25: 4298-4307.

10. Karkkainen MJ, Petrova TV. Vascular endothelial growth factor receptors in the regulation of angiogenesis and lymphangiogenesis. Oncogene 2000; 19: 5598-5605.

11. Alitalo K, Mohla S, Ruoslahti E. Lymphangiogenesis and cancer: meeting report. Cancer Res 2004; 64: 9225-9229.

12. Schoppmann SF, Horvat R, Birner P. Lymphatic vessels and lymphangiogenesis in female cancer: mechanisms, clinical impact and possible implications for anti-lymphangiogenic therapies (Review). Oncol Rep 2002; 9: 455-460

13. Niki T, Iba S, Tokunou M, et al. Expression of vascular endothelial growth factors $A, B, C$, and $D$ and their relationships to lymph node status in lung adenocarcinoma. Clin Cancer Res 2000; 6: 2431-2439.

14. Branca M, Ciotti M, Giorgi C, et al. Predicting high-risk human papillomavirus infection, progression of cervical intraepithelial neoplasia, and prognosis of cervical cancer with a panel of 13 biomarkers tested in multivariate modeling. Int J Gynecol Pathol 2008; 27: 265-273.

15. Tian X, Cong M, Zhou W, et al. Relationship between protein expression of VEGF-C, MMP-2 and lymph node metastasis in papillary thyroid cancer. J Int Med Res 2008; 36: 699-703.

16. Ma D, Xu Y, Zhu L. Expression of vascular endothelial growth factor $\mathrm{C}$ correlates with a poor prognosis based on analysis of prognostic factors in patients with cervical carcinoma. J Obstet Gynaecol Res 2011 37: 1519-1524.

17. Sotiropoulou N, Bravou V, Kounelis S, et al. Tumour expression of lymphangiogenic growth factors but not lymphatic vessel density is implicated in human cervical cancer progression. Pathology 2010; 42: 629-636.

18. Jach R, Dulinska-Litewka J, Laidler P, et al. Expression of VEGF, VEGF-C and VEGFR-2 in in situ and invasive SCC of cervix. Front Biosci 2010; 2: 411-423.

19. Mitsuhashi A, Suzuka K, Matsui H, et al. Serum vascular endothelial growth factor (VEGF) and VEGF-C levels as tumor markers in atients with cervical carcinoma. Cancer 2005; 103: 724-730.
20. Hashimoto I, Kodama J, Seki N, et al. Vascular endothelial growth factorC expression and its relationship to pelvic lymph node status in invasive cervical cancer. Br J Cancer 2001; 85: 93-97.

21. Yokoyama Y, Charnock-Jones DS, Licence D, et al. Expression of vascular endothelial growth factor (VEGF)-D and its receptor, VEGF receptor 3, as a prognostic factor in endometrial carcinoma. Clin Cancer Res 2003; 9: 1361-1369.

22. Yong S, Adnane J, Trail P, et al. Sorafenib (BAY 43-9006) inhibits tumor growth and vascularisation and induces tumor apoptosis and hypoxia in RCC xenograft models. Cancer Chem Pharmacol 2007; 59: 561-574.

23. Nakamura Y, Yasuoka Y, Tsujimoto M, et al. Prognostic significance of vascular endothelial growth factor $D$ in breast carcinoma with longterm follow-up. Clin Cancer Res 2003; 9: 716-721.

24. Gunningham SP, Currie MJ, Han C, et al. The short form of the alternatively spliced flt-4 but not its ligand vascular endothelial growth factor $C$ is related to lymph node metastasis in human breast cancers. Clin Cancer Res 2000; 6: 4278-4286.

25. Jacquemier J, Mathoulin-Portier MP, Vatola R, et al. Prognosis of breastcarcinoma lymphagenesis evaluated by immunohistochemical investigation of vascular-endothelial-growth-factor receptor 3. Int J Cancer 2000; 89: 69-73.

26. Shi X, Chen G, Xing H, et al. VEGF-C, VEGFR-3 and COX-2 enhances growth and methastasis of human cervical carcinoma cell lines in vitro. Oncol Rep 2007; 18: 241-247.

27. Chaudary N, Milosevic M, Hill RP. Suppression of vascular endothelial growth factor receptor 3 (VEGFR3) and vascular endothelial growth factor C (VEGFC) inhibits hypoxia-induced lymph node metastases in cervix cancer. Gynecol Ocol 2011; 123: 393-400.

28. Mian H, Yang C, Wen L, et al. Vascular endothelial growth factor C promotes cervical cancer metastasis via up-regulation and activation of RhoA/ROCK-2/moesin cascade. BMC Cancer 2010; 10: 170-182.

29. Xiaoyan S, Gang C, Hui X, et al. VEGF-C, VEGFR-3, and COX-2 enhances growth and metastasis of human cervical carcinoma cell lines in vitro. Oncol Reports 2007; 18: 241-247.

30. Van Trappen PO, Steele D, Lowe DG, et al. Expression of vascular endothelialgrowth factor (VEGF)-C and VEGF-D, and their receptor VEGFR-3 during different stages of cervical carcinogenesis. J Pathol 2003; 201: 544-554. 